\title{
On the role of the entorhinal cortex in the effective connectivity of the hippocampal formation
}

\author{
Víctor J. López-Madrona, ${ }^{1}$ Fernanda S. Matias, ${ }^{2}$ Ernesto Pereda, ${ }^{3}$ Santiago Canals, ${ }^{1}$ \\ and Claudio R. Mirasso ${ }^{4}$ \\ ${ }^{1}$ Instituto de Neurociencias, Consejo Superior de Investigaciones Científicas, Universidad Miguel Hernández, \\ Sant Joan d'Alacant 03550, Spain \\ ${ }^{2}$ Instituto de Física, Universidade Federal de Alagoas, Maceió, Alagoas 57072-970, Brazil \\ ${ }^{3}$ Departamento de Ingeniería Industrial, Escuela Superior de Ingeniería y Tecnología \& Instituto \\ Universitario de Neurociencia, Universidad de La Laguna, Avda. Astrofísico Fco. Sánchez, s/n, La Laguna, \\ Tenerife 38205, Spain \\ ${ }^{4}$ Instituto de Fisica Interdisciplinar y Sistemas Complejos, IFISC, CSIC-UIB, Campus Universitat de les Illes \\ Balears, E-07122 Palma de Mallorca, Spain
}

(Received 23 October 2016; accepted 10 January 2017; published online 31 March 2017)

\begin{abstract}
Inferring effective connectivity from neurophysiological data is a challenging task. In particular, only a finite (and usually small) number of sites are simultaneously recorded, while the response of one of these sites can be influenced by other sites that are not being recorded. In the hippocampal formation, for instance, the connections between areas CA1-CA3, the dentate gyrus (DG), and the entorhinal cortex (EC) are well established. However, little is known about the relations within the EC layers, which might strongly affect the resulting effective connectivity estimations. In this work, we build excitatory/inhibitory neuronal populations representing the four areas CA1, CA3, the DG, and the EC and fix their connectivities. We model the EC by three layers (LII, LIII, and LV) and assume any possible connection between them. Our results, based on Granger Causality (GC) and Partial Transfer Entropy (PTE) measurements, reveal that the estimation of effective connectivity in the hippocampus strongly depends on the connectivities between EC layers. Moreover, we find, for certain EC configurations, very different results when comparing GC and PTE measurements. We further demonstrate that causal links can be robustly inferred regardless of the excitatory or inhibitory nature of the connection, adding complexity to their interpretation. Overall, our work highlights the importance of a careful analysis of the connectivity methods to prevent unrealistic conclusions when only partial information about the experimental system is available, as usually happens in brain networks. Our results suggest that the combination of causality measures with neuronal modeling based on precise neuroanatomical tracing may provide a powerful framework to disambiguate causal interactions in the brain. Published by AIP Publishing. [http://dx.doi.org/10.1063/1.4979001]
\end{abstract}

Finding a relation between structural and functional brain connectivity is one of the major goals of neuroscientists. In order to tackle this issue, two questions can be addressed: How do anatomical connections among brain regions influence the information flow in the brain network? And how to determine the structural connectivity from the effective connectivity (or, more precisely, from causal measurements)? In this work, we model the hippocampal formation assuming three main areas (CA1, CA3, and the dentate gyrus) and the entorhinal cortex (EC), with the aim of exploring how the structural connectivity between EC layers influences the causal links among these three hippocampal regions. We apply two different statistical causality measurements to the simulated neurophysiological time series: Granger Causality (GC) and Partial Transfer Entropy (PTE). Our results reveal that the estimation of effective connectivity in the hippocampus strongly depends on the connectivity among EC layers. We also find that GC and PTE may yield very different results for certain configurations among the layers of the $\mathrm{EC}$. In addition, we show that based on GC and PTE measurements, it is not possible to differentiate between excitatory and inhibitory inter-areal connections.

\section{INTRODUCTION}

One of the most challenging tasks in systems neuroscience is to understand how the relatively stable anatomical structure of the brain can generate the large diversity of functional states observed when recording its activity. ${ }^{1}$ A number of processes operating at different time- and spatial-scales have been demonstrated to dynamically modify the coupling between the anatomically embedded, and otherwise static, brain elements. At single synapse scales, the strength and efficacy of neurotransmission between neurons are tightly regulated by short- and long-term synaptic plasticity processes that rely on the frequency and intensity of a particular synapse, as well as its history of use. ${ }^{2-4}$ At the cellular level, membrane excitability, regulated by the balance between excitatory and inhibitory inputs or cell-autonomous processes determining the densities of ionic channels, marks 
which neurons participate in active ensembles, in particular, functional states. ${ }^{5-8}$ The levels of neuromodulatory neurotransmission regulate, in contrast, the activity of populations of neurons instead of single synapses or neurons and do so in response to changes in the internal (i.e., metabolic) or external environment. ${ }^{9-11}$ In this scenario, even the rich anatomical information used in some cases to reconstruct quite complete wiring diagrams of small portions of the nervous system ${ }^{12,13}$ will most likely be insufficient to derive comparably precise and comprehensive functional diagrams incorporating causal or effective interactions between the brain network elements.

In the present work, we use a computational model of the hippocampal formation to explore the capacity of linear and nonlinear measurements of directed statistical interdependencies between simulated neurophysiological time series to retrieve effective connections between the simulated populations and draw complete functional diagrams. The hippocampal formation contains the hippocampus proper (CA1-CA3 regions), the dentate gyrus (DG), and the entorhinal cortex $(\mathrm{EC})^{14-16}$ (see Figure 1). In this system, two parallel circuits have been described. One is the so called trisynaptic circuit, which is composed by EC layer II projections to the DG, which relay activity to $\mathrm{CA} 3$ through the mossy fibers and then, $\mathrm{CA} 3$ neurons project to $\mathrm{CA} 1$ ( $\mathrm{LII} \rightarrow \mathrm{DG} \rightarrow \mathrm{CA} 3 \rightarrow \mathrm{CA} 1$ ) to finally reenter in the deep layers of EC. In this circuit CA3, neurons also receive a direct projection from $\mathrm{EC}$ layer II. The other circuit is the so called temporoanatomic circuit, in which CA1 neurons receive a direct projection from EC layer III (LIII $\rightarrow \mathrm{CA} 1$ ). These different regions and circuits have been proposed to contribute to different aspects of learning and memory by modeling work. ${ }^{17-19}$ However, while the main excitatory connections between and within the DG and hippocampus proper have been worked out in detail, the inhibitory components and the intrinsic EC connections are comparatively less understood. Here we analyze, using Granger Causality (GC) and Transfer Entropy (TE) measurements, the effective connectivity among the DG, CA3, and CA1 while systematically modifying the internal connectivity in the EC and introducing an inhibitory connection in the hippocampus proper. We aim at understanding whether (and how) the results from these measures as applied to hippocampal signals depend on the connections within the EC, so that such results can be used to gain insight into the EC connectivity structure.

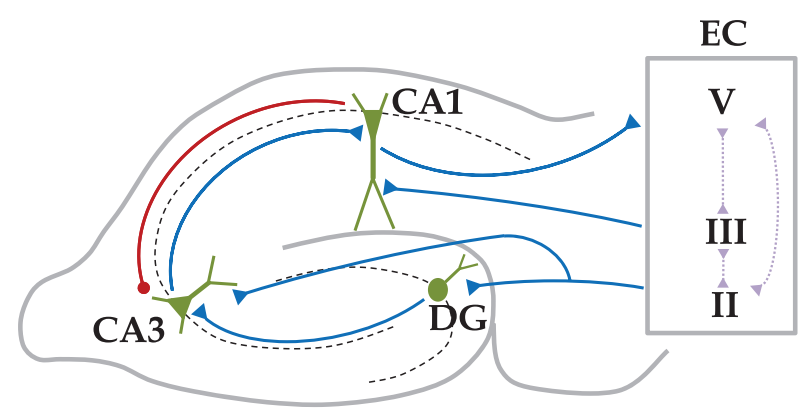

FIG. 1. Circuit. Schematic representation of the hippocampus circuit. The connectivity between the three main areas is kept constant while that between the different layers in the entorhinal cortex is changed.

\section{MODEL}

As said above, we consider our circuit model composed by the regions $\mathrm{CA} 1, \mathrm{CA} 3, \mathrm{DG}$, and the $\mathrm{EC}$ with three main layers. LII connects to DG and CA3, LIII connects to CA1, and LV receives reentrant inputs from CA1. It is known that these areas have different spiking behaviors (see, e.g., chapter 8 of Ref. 20). For instance, stellate neurons of the EC combine subthreshold oscillations with intervals of regular spikes, depending on the injected current. CA1 and CA3 areas mainly contain pyramidal neurons that exhibit subthreshold oscillations and fire regular spikes or bursts depending on the injected current. The granule cells of the DG fire very sparsely with prolonged silent periods and occasionally in bursts; they also display subthreshold oscillations. Considering this variety of behaviors is crucial in some cases, e.g., when studying synchronization aspects. However, our aim in this work is to study information flow and the results should not depend much on the intrinsic dynamics of the particular region but on the dominance of one area over the other when information flows in a given circuit. For this reason, each area, as well as the EC layers, are described by a simple population of sparsely connected excitatory and inhibitory neurons. Each population is connected with other populations of the circuit as shown in Figure 1. All excitatory connections between the structures are kept constant, but for the EC layers, connections are systematically modified to investigate their effect on the hippocampal effective connectivity. Inhibitory connections are introduced between CA1 and CA3, and their influence on overall connectivity is also investigated. From a purely anatomical perspective, the implemented inhibitory connection is located between the two hippocampal associative loops that start from EC Layers II and III, respectively. Therefore, both information channels can be mixed either through interconnection in the EC or by the introduced inhibitory link. This rather simple configuration allowed us to confront current causality measures with an ambiguous situation in which a causal interaction can be found due to feed forward excitatory activity propagation (information transmission) or backward inhibition (synchronization of neuronal populations). In what follows, we describe the model we use for the populations and connections and give an insight into the definitions of the GC and TE.

\section{A. Neuronal populations}

Each area and each layer shown in Fig. 1 are modeled as a neuronal population composed of 400 excitatory and 100 inhibitory neurons described by the Izhikevich model. ${ }^{21}$ Each neuron receives 50 synapses (sparse connectivity $\approx 10 \%$ ) from randomly selected neighbors (excitatory or inhibitory) in the same population. For the coupling between the different areas, either excitatory or inhibitory, we assume that each postsynaptic neuron receives 20 synapses from presynaptic neurons in the sender population. The membrane potential $v$ and the recovery variable $u$, that accounts for the activation of $\mathrm{K}^{+}$and inactivation of $\mathrm{Na}^{+}$, of each neuron are described by 


$$
\begin{gathered}
\frac{d v}{d t}=0.04 v^{2}+5 v+140-u+\sum I_{x}, \\
\frac{d u}{d t}=a(b v-u) .
\end{gathered}
$$

The summation $\sum I_{x}$ is over all the synaptic currents. The model establishes that when $v \geq 30 \mathrm{mV}$, its value is reset to $c$ and $u$ is reset to $u+d$. Following Ref. 21, for each excitatory neuron $(a, b)=(0.02,0.2)$ and $(c, d)=(-65,8)+(15$, $-6) \sigma^{2}$, whereas for each inhibitory neuron $(a, b)=(0.02$, $0.25)+(0.08,-0.05) \sigma$ and $(c, d)=(-65,2) . \sigma$ is a random variable uniformly distributed on the interval $[0,1]$ and determines the proportion of different spiking neurons (between regular spiking to bursting modes).

The synaptic currents $I_{x}$, which can be excitatory and inhibitory mediated by $\operatorname{AMPA}(A)$ and $\operatorname{GABA}_{A}(G)$, respectively, are described by the following equations:

$$
I_{x}=g_{x} r_{x}\left(E_{x}-v\right),
$$

where $x=A, G$ and $E_{A}=0 \mathrm{mV}$ and $E_{G}=-65 \mathrm{mV}$ are the reversal potentials. Unless otherwise stated all excitatory (inhibitory) weights are set to $g_{A}=0.5 \mathrm{~ms}\left(g_{G}=2 \mathrm{nS}\right)$. The dynamics of the fraction of bound synaptic receptors $r_{x}$ is given by

$$
\tau_{x} \frac{d r_{x}}{d t}=-r_{x}+D \sum_{k} \delta\left(t-t_{k}\right) .
$$

The summation over $k$ stands for pre-synaptic neurons. D is taken, without loss of generality, equal to 0.05 . The time decays are taken as $\tau_{A}=5.26 \mathrm{~ms}$ and $\tau_{G}=5.6 \mathrm{~ms}$. Each neuron is subject to an independent Poisson input, representing $n$ pre-synaptic neurons, not included in the populations, from other regions spiking with rate $R / n$. The rate $R$ strongly influences the rhythm of the population. To mimic the experimentally measured activity of the hippocampus, we take $R=600 \mathrm{~Hz}$ (see Figure 2). The Poissonian synapses are assumed as excitatory (AMPA) connections. The analyzed time series correspond to the mean membrane potential of
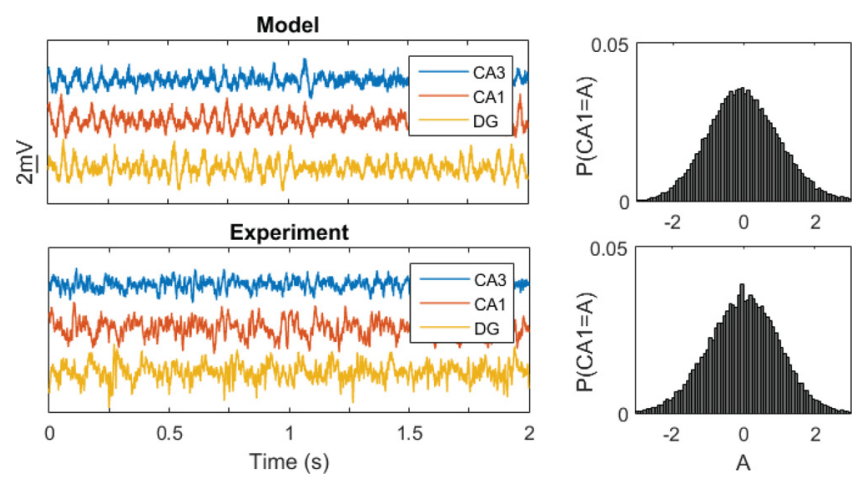

FIG. 2. Representative time series. Upper panel: Left, examples of the temporal evolution of the mean membrane potential, as a crude approximation of the experimentally measured local field potential (LFP), obtained from all neurons in a specific population, CA1, CA3, and DG. Right, normalized probability distribution function obtained from the numerical time traces for area CA1. Lower panel: same as upper panels but from experimental data. Adapted from Ref. 23. each population, which is calculated as the average value of $v$ for all neurons within the population. ${ }^{22}$

For each neuronal population, we generate one time series with a duration of $24 \mathrm{~s}$ using a sampling of $F s=20 \mathrm{kHz}$. We discard the first $4 \mathrm{~s}$ to eliminate transient states, until the signal is covariance stationary, i.e., with constant mean and variance. Once this regime is reached, the time series are down sampled at $250 \mathrm{~Hz}$ for the computation analysis. In Fig. 2, we show an example of time traces generated with the model and recorded in experiments on rats. ${ }^{23}$ It can be seen that both traces are quite similar in terms of normalized probability distribution function, so causal measurements based on entropy like PTE will have the same efficiency in our model than in real data.

\section{B. Effective connectivity}

The study of the relationship between different neuronal oscillators/brain areas from the signals they produce entails the assessment of the statistical dependence between these signals. This statistical dependence is often termed functional connectivity (FC). Since FC refers to the functional relationship between spatially separated brain areas/neural populations, it does not distinguish directional influence or if the activities of the regions are due to a common driver. The existence of directionality in this statistical dependence, whereby one area influences the other or vice versa, is referred as effective connectivity. Different time series methods (bi- and multivariate) exist, which are able to assess the existence of effective connectivity between two or more signals. In the following, we describe the two methods that we use in this work.

\section{Granger causality}

The concept of GC was published by Granger almost fifty years ago. ${ }^{24}$ Nowadays, it continues to be one of the most used methods to study directionality in the functional connectivity of time series. The basic idea is quite straightforward. Let $X(t)$ and $Y(t)$ be two given time series. The autoregressive model for each of the signals is constructed by assigning to each variable its $p$ last samples plus a residual term. This is written as

$$
\begin{aligned}
& X(t)=\sum_{i=1}^{p} A_{1 i} X(t-i)+\varepsilon_{1}(t) \\
& Y(t)=\sum_{i=1}^{p} A_{2 i} Y(t-i)+\varepsilon_{2}(t) .
\end{aligned}
$$

The constant matrix $A$ contains the coefficients that determine the information from the present state of both signals that can be extracted from their own past. The model order $p$ is estimated using some appropriate information criteria such as Akaike's (AIC) ${ }^{25}$ to prevent overfitting. If one adds to the model the past values of one of the variables (e.g., $Y$ ) to predict the other one (e.g., $X$ ) and vice versa, the following bivariate autoregressive model of the signals is obtained: 


$$
\begin{aligned}
& X(t)=\sum_{i=1}^{p} A_{11 i} X(t-i)+\sum_{i=1}^{p} A_{12 i} Y(t-i)+\mu_{1}(t) \\
& Y(t)=\sum_{i=1}^{p} A_{21 i} X(t-i)+\sum_{i=1}^{p} A_{22 i} Y(t-i)+\mu_{2}(t) .
\end{aligned}
$$

If, for example, the variance of the bivariate model $\operatorname{var}\left(\mu_{1}\right)$ is smaller than that of the univariate one $\operatorname{var}\left(\varepsilon_{1}\right)$, it implies that the past of $Y(t)$ improves the prediction of $X(t)$; or, in other words, $Y$ G-causes $X$.

Generally, we deal with more than two time series. For these cases, it is easy to generalize the bivariate model to a more general, multivariate autoregressive (MVAR) model. In the MVAR, we can test if $Y$ G-causes $X$ when the effect of a set of different time series $Z_{1} \ldots Z_{n}$, which are included in the model, is taken into account. ${ }^{26}$ Thereby, we can differentiate if $Y(t)$ directly G-causes $X(t)$ or if their relationship is an indirect one, mediated by other variable $Z$. If this happens, we say that $Y$ G-causes $Z$, which G-causes $X$. This allows distinguishing direct from indirect connections provided all external variables mediating the relationship between $X(t)$ and $Y(t)$ have been included in the MVAR model. The statistical significance is measured via an $F$-test on the null hypothesis (p-value 0.01 ) to check that $A_{i j}$ are zero, corrected by the Bonferroni correction. ${ }^{27}$ In this work, we use the GCCA MATLAB toolbox ${ }^{27}$ to compute Granger causalities.

\section{Transfer entropy}

One fundamental limitation of the GC measurement is that the autoregressive models are linear, so that this index is insensitive to non-linear interactions between the signals. Different implementations have been suggested to overcome this limitation, ${ }^{28-30}$ yet none of them turned out to be completely satisfactory. Instead, the $\mathrm{TE}^{31}$ is an index based on a very similar concept to that of the GC but using conditional entropies rather than MVAR models. This makes TE sensitive also to nonlinear interactions. Thus, given two signals $X(t)$ and $Y(t), T E_{X \rightarrow Y}$ is defined as:

$$
\begin{aligned}
T E_{X \rightarrow Y}= & H\left(Y_{t} \mid Y_{t-1: t-\tau}\right) \\
& -H\left(Y_{t} \mid Y_{t-1: t-\tau}, X_{t-1: t-\tau}\right),
\end{aligned}
$$

where $H()$ denotes the entropy and $\tau$ is a temporal delay. $T E_{X \rightarrow Y}$ measures the reduction in entropy on the state of one variable $Y(t)$ when the past of the second variable $X(t)$ is included alongside the past of $Y(t)$. Moreover, a more accurate measurement exists, called Partial Transfer Entropy (PTE), that allows us to differentiate between direct links and those mediated by intermediate nodes. Its definition results from adding variables to the conditional entropies defined in (7), such that the PTE takes the form

$$
\begin{aligned}
P T E_{X \rightarrow Y, Z}= & H\left(Y_{t} \mid Y_{t-1: t-\tau}, Z_{t-1: t-\tau}\right) \\
& -H\left(Y_{t} \mid Y_{t-1: t-\tau}, Z_{t-1: t-\tau}, X_{t-1: t-\tau}\right) .
\end{aligned}
$$

Here, $Z_{t}=\left\{Z_{1, t}, Z_{2, t}, \ldots, Z_{n, t}\right\}$ is a multivariate set of $n$ random variables. The PTE measures the influence of the past of $X(t)$ on the present of $Y(t)$ that cannot be accounted for by the past of $Z$. Thus, it is possible to estimate the information directly transferred from $X(t)$ to $Y(t)$ (or vice versa), without the contribution of any other (measured) variable. To compute the PTE, we use the HERMES MATLAB toolbox. ${ }^{32}$ Finally, we assess the statistical significance of both GC and PTE by constructing, in each case, 100 surrogate data sharing most of the linear properties of the original signals but being independent from each other. For this purpose, we used the iterative amplitude adjusted Fourier transform surrogate method ${ }^{33}$ as implemented in HERMES. Effective connectivity between $X(t)$ and $Y(t)$ was considered significant at the $p<0.01$ level (non-parametric rank test), if the corresponding index (GC or PTE) was greater for the original data than for any of the 100 surrogate pairs.

\section{RESULTS}

\section{A. Simple motif with interneuron}

In general, causality between two nodes is assumed as an excitatory driver-driven relationship: if after an event that occurs in a node A there is always another event in node B, then we assume a causal influence from A to B. In other words, we say that information flows from A to B. However, this is not the only possible type of relationship between two connected neurons and neuronal populations. For instance, it might happen that a node A projects an inhibitory connection to a node B causing, e.g., B to stop firing. In this case, the causal effect still exists due to the influence from A to B but the meaning is different to the one mentioned above. In the first case, the effective influence can be interpreted as direct information transmission from $\mathrm{A}$ to $\mathrm{B}$, while in the second case $\mathrm{A}$ imposes the timing (rhythmicity) at which $\mathrm{B}$ can be sending information. Yet, in both cases, the existence of a cause-effect relationship should be detected by either the (P)TE and/or the GC.

To check whether this is indeed the case, we consider a very simple model of the connections within the hippocampus and from the EC to the hippocampus (Figure 3(a)). This reduced circuit includes only four populations: two of them are nodes of the hippocampus (CA1 and CA3) and the remaining two belong to the EC (layers II and III). Excitatory links are included from layers II and III to CA3 and $\mathrm{CA} 1$, respectively, together with an excitatory connection from CA3 to CA1, which is part of the well-known trisynaptic pathway. We also add an inhibitory connection from CA1 to CA3, which represents the projection of an inhibitory subpopulation in CA1 projecting back to CA3. ${ }^{34}$ The causal analysis, with both GC and PTE, correctly identifies all the excitatory and inhibitory links of the model and the direction of each interaction, as can be seen in Figures 3(b) and 3(c). However, also as expected, we cannot differentiate the type of connection, namely, whether it is excitatory or inhibitory. This is so regardless of the type of connection. In both cases the past of one of the signals conditions the present of the other one. 
A

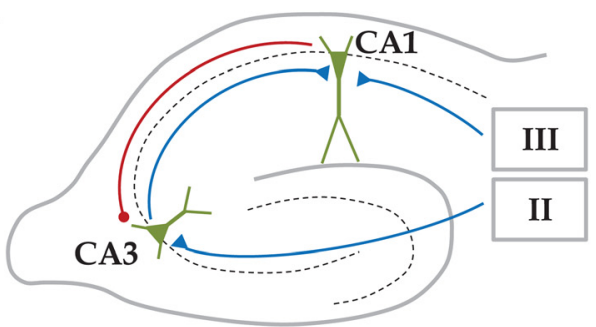

C

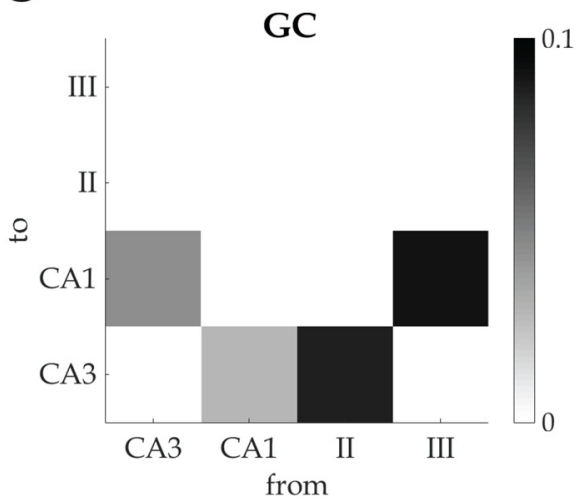

B

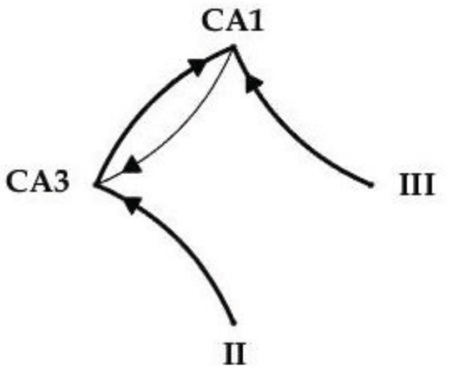

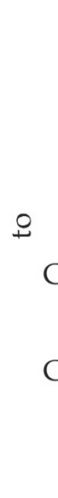

\section{B. Incomplete circuit}

The brain is not just a tangle of wires linking each area with all others but, like in a motherboard, it has different structures with specific functions that are sparsely interconnected to optimize the performance. This allows us to analyze each region separately and use causality measures to estimate the internal connectivity patterns of each of these units. Yet, as we said, each brain structure is connected to a number of other areas. Therefore, its activity depends not only on its internal structure but also, in a non-trivial way, on the state of those areas to which it is connected. An example, in our case, is the relationship between the hippocampus proper and DG with the EC. Each of these structures has its own local circuitry, while at the same time, they are connected to each other. During the process of memory encoding and retrieval, information travels through these areas forming inter-region associative loops. Any causal analysis of effective connectivity in the hippocampus requires, therefore, the consideration of the whole circuit (including the EC layers).

To better understand how the internal wiring of the EC influences the results of a causal analysis in the other hippocampal formation structures, we built a model in which all connections but those of the EC are fixed (see methods). We also considered inhibition between CA1 and CA3 to further explore the contribution of inhibitory links on causality measurements. In what follows, we analyze how the internal connectivity between the three layers of the EC affects the results obtained by $\mathrm{GC}$ and $\mathrm{TE}$ analysis of the hippocampus when only measures on DG, CA3, and CA1 nodes are available, as commonly happens experimentally.

\section{Connections between the layers of the entorhinal cortex}

As mentioned before, in our connectivity analysis we only consider the signals generated by DG, CA3, and CA1 and calculate GC and PTE assuming different internal connections between the layers of the EC. As we consider three EC layers, each configuration is given by the presence or absence of six links (II $\rightarrow$ III, III $\rightarrow$ II, II $\rightarrow$ V, V $\rightarrow$ II, III $\rightarrow$ V, and V $\rightarrow$ III), with a total of $2^{6}=64$ possibilities. To represent all combinations in a square matrix, we consider the state of three links (II $\rightarrow \mathrm{V}, \mathrm{V} \rightarrow \mathrm{II}$, and $\mathrm{V} \rightarrow \mathrm{III}$ ) in each column, and the other connections (II $\rightarrow \mathrm{III}$, III $\rightarrow \mathrm{II}$, and $\mathrm{III} \rightarrow \mathrm{V}$ ) in each row. In Fig. 4, we show the results for all possible combinations of the internal EC connections, where each box represents the connectivity estimated by GC (left) and PTE (right) between the three populations $(1=\mathrm{CA} 3,2=\mathrm{CA} 1$, and $3=\mathrm{DG})$. The interconnections in the EC for each box can be obtained by adding up the links between layers in the corresponding row and column. For example, in row 2, column 3, we have the causality results in the hippocampus when layers II and III project to layer V. Although we are aware of the unlikeliness of some of the resulting EC connectivity configurations, based on the existing literature, ${ }^{16}$ we present all of them for completeness and highlight later some specific cases.

We start by checking whether different connections between EC layers result in different connectivity maps in the hippocampus. The GC and PTE measurements shown in the figure demonstrate that this is indeed the case. Importantly, discrepancies are not only found between different EC wiring diagrams but also between the two methods to estimate causality for the same EC scheme. There are two main factors known to affect causality measurements. On the one hand, in a chain of unidirectional links $(\mathrm{A} \rightarrow \mathrm{B} \rightarrow \mathrm{C})$, in the absence of the intermediate node $(\mathrm{B})$ in the analysis, a direct causality between the adjacent nodes $(\mathrm{A} \rightarrow \mathrm{C})$ is found. On the other hand, if a node A projects simultaneously to two nodes, $\mathrm{B}$ and $\mathrm{C}(\mathrm{B} \leftarrow \mathrm{A} \rightarrow \mathrm{C})$, but $\mathrm{A}$ is not included in the analysis, a misleading causal relationship ( $\mathrm{B} \leftrightarrow \mathrm{C}$ ) can be expected. This is due to the fact that nodes $\mathrm{B}$ and $\mathrm{C}$ share the same information, which cannot be removed due to a lack of partialization. The 


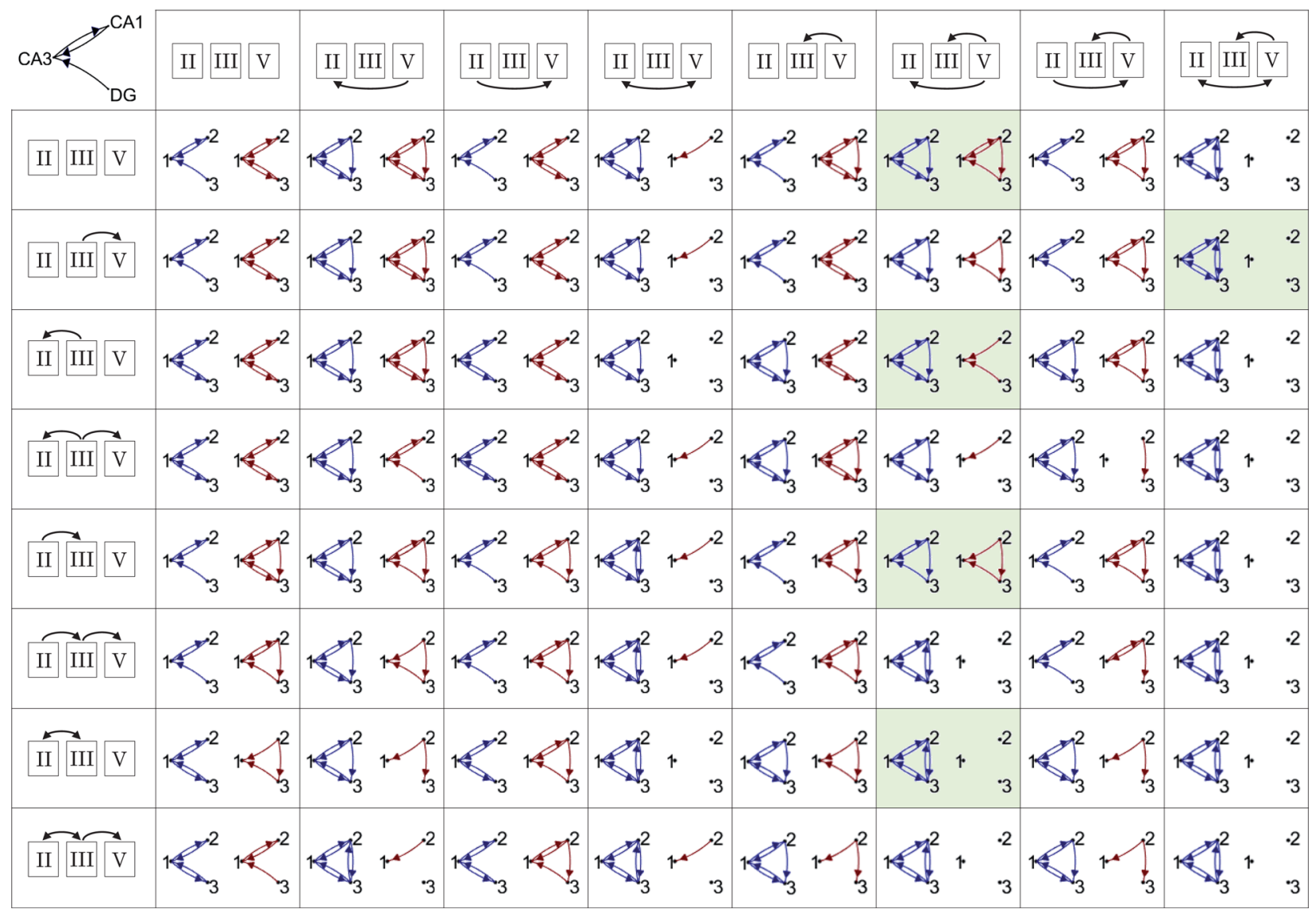

FIG. 4. Effective connectivity. Results of the GC (blue) and PTE (red) measurements for all possible combinations of unidirectional or bidirectional connections between EC layers II, III, and V. Highlighted boxes represent most relevant motifs. (Upper left corner) Links implemented in the model.

first example is found in our results in row 1 , column 6 , where due to the projections from EC layer V to layers II and III, a loop is generated with the information traveling from CA1 to EC and coming back to the hippocampus after passing through all layers. Therefore, both GC and PTE measurements reveal a causal influence from CA1 to the DG, layers II and V being the intermediate links in the chain. Indeed, since CA1 is part of the trisynaptic circuit receiving information from DG through CA3, a connection from LV to LII is sufficient to create the associative loop between circuits and lead to the misleading conclusion, based on causal measurements, of a direct $\mathrm{CA} 1 \rightarrow$ DG connection. The second example can be found in row 1 , column 1 , where $\mathrm{CA} 3$ and $\mathrm{DG}$ populations receive information from layer II. Since the EC nodes are not included in the causality calculations, PTE finds a link from CA3 to the DG which is not present in our neuronal model. This spurious link does not appear when computing the GC. This result might suggest that GC measurements are more robust than PTE measurements in these situations.

If we compare the GC and PTE methods, it is easy to see in Fig. 4 two different groups of results. Under simple configurations of the EC layers, the connectivity schemes are quite similar, especially in those cases where layer $\mathrm{V}$ has no links to the other layers (loops are avoided). On the contrary, if we impose loops in the EC, the two methods give very different results (see, e.g., column 8). This might be due to the fact that there are many ways in which a signal from one node reaches other nodes. Moreover, this signal could arrive to a certain node more than once and at different time lags, yielding distinct results for separate time windows. The causality obtained for this model with GC gives a scheme with all populations connected, while PTE does not find any significant link.

Note that, theoretically, PTE should detect interdependency in cases where multivariate GC does not, because the latter one is based in MVAR models and therefore only sensitive to linear interdependencies, whereas PTE is sensitive to both linear and nonlinear interdependencies. Yet we found sometimes that GC between two nodes is significant whereas the PTE is not. We believe that the more likely explanation for this seemingly counter-intuitive result is that PTE is able to efficiently detect when the connectivity between any two areas in the model is direct rather than indirect, i.e., the information on 1 which can be extracted from 2 is not also present in 3, whereas the GC incorrectly detects these indirect connections when the common information is not included in the partializing set. This hypothesis is supported also by the fact that those cases where the discrepancies are more apparent (i.e., fourth and last columns from the left in Fig. 4) correspond to cases with a bilateral connection between layers LII and LV in the EC, which in out model project to DG and CA1, respectively. This shared information seems to be detected by the PTE, and mask, if any, the true direct one, whereas this is not the case for the GC. Either way, the 
conclusion remains that without including the whole circuitry (EC and hippocampus) in the analysis, the results based only on portion of it can be misleading depending on the connections within the unobserved areas.

The connectivity model in the EC has been kept intentionally simple, and this has likely conditioned the results. This decision was in part due to the lack of detailed information on the interactions between EC layers as well as the reduced information on the inhibitory connections. Although a model with inhibitory connections within the EC and between regions could give rise to more realistic associative circuits in the hippocampal formation, in light of our present results it is not clear that GC and PTE measurements could reconstruct them correctly from a partial set of activity recordings (i.e., DG, CA3 and CA1), and, in any case, it is out of the scope of the present paper.

\section{Most relevant motifs}

In Fig. 4, we analyze all the possible excitatory interconnection schemes within 3 layers of EC. However, not all these schemes are equally significant from a neurobiological point of view. In this section, we focus on those patterns of internal EC connectivity that find support in the current neuroanatomical literature. More specifically, connections from LV to LII and LIII have been consistently demonstrated in different animal species, and interconnections between LII and LIII, although less studied, have been also described. ${ }^{16}$

\section{1. $L V \rightarrow L I I+L V \rightarrow L I I I$}

This connection confers the system a characteristic loop that allows the re-entrance on information into the EC that has been processed previously in the hippocampus. This functional loop has been demonstrated experimentally ${ }^{35}$ and is proposed to form an associative memory system with capacities for encoding and retrieval. ${ }^{36}$ As indicated above, this connection scheme poses a challenge on causality techniques to disambiguate the correct interdependencies. More specifically in our model, DG and CA1 may communicate either through CA3 or through the EC. Two direct connections not introduced in the model are however highlighted by the causality analysis: A direct projection from CA1 to DG and a backprojection from CA3 to DG. In Fig. 5, we show the role that inhibition plays in these results, with a causal analysis of the same model but without including the inhibitory link from CA1 to CA3. The two methods, GC and PTE, render the same connectivity between $\mathrm{CA} 1$ and $\mathrm{CA} 3$, demonstrating that this effective connection is equally established through direct inhibition or indirect excitation mediated by the EC. However, the spurious connection from CA1 to the DG estimated by the PTE in the presence of an inhibitory link is disambiguated after inhibitory removal. This result highlights remote cascading effects associated with long-range interareal inhibition.

\section{2. $L V \rightarrow L I I+L V \rightarrow L I I I+L I I I \rightarrow L I I$}

When a projection from layer III to layer II is added to the connections assumed in the previous paragraph, we close
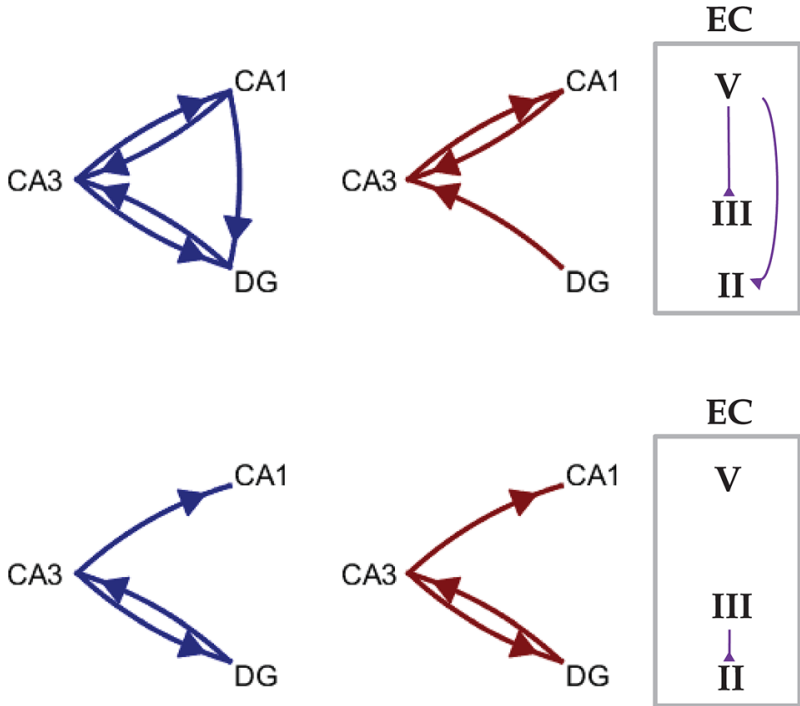

FIG. 5. Causality without inhibition. Top: GC and PTE measurements in blue and red, respectively, for our original model without the inhibition link from CA1 to CA3 and an EC scheme with layer V projecting to layers II and III. Bottom: same measurements for an EC scheme with a connectivity from layer III to layer II. Eliminating the inhibitory link only removes the causality from CA1 to CA3.

a loop in the EC. In these situations, the information in layer III will arrive first to CA1 and then to DG and CA3 through layer II. Accordingly, the GC analysis finds direct connections from CA1 to these structures, while PTE is more restrictive, revealing only connections from the DG and CA1 to CA3 (see row 3 column 6 in Fig. 4).

\section{3. $L V \rightarrow L I I+L V \rightarrow L I I I+L I I \rightarrow L I I I$}

A projection from layer II to layer III would result in common inputs to CA3, DG, and CA1. However, since anatomical connection from CA3 and DG to CA1 is considered in the model and activity from these three nodes is known, the LII-LIII connection should not modify the effective connectivity derived from the causality analysis. The PTE calculations, which give similar results to the GC except for the link from $\mathrm{CA} 3$ to $\mathrm{CA} 1$, show that our hypothesis is indeed correct (see row 5 column 6 in Fig. 4).

\section{4. $L V \rightarrow L I I+L V \rightarrow L I I I+L I I \leftrightarrow L I I I L V \leftrightarrow L I I+L V \leftrightarrow L I I I$}

In both cases, the complexity of the EC internal structure not only prevents us to find any kind of causality from the information measured in the hippocampus but also yields different results with both methods. This is because of all the possible ways the information can flow in the circuits which are not taken into account in the model. "This illustrates, as mentioned before, some limitations of the causal analysis. When the activity of critically connected nodes in the circuit is not included in the analysis, the calculations of information flow are drastically affected, yielding unrealistic results (see, e.g., row 7 column 6 and row 2 column 8 in Fig. 4)." 


\section{CONCLUDING REMARKS}

We have performed a causal analysis of the information flow in a reduced circuit of the hippocampus containing areas CA1, CA3, and the DG. For the computation of GC and PTE measurements, we assumed that the activity of only these three areas was available, as occurs in many experimental conditions. In the full model, however, we also incorporated the EC layers II, III, and V, which are known to be essential for hippocampal function and introduce distinct associative loops between hippocampal inputs and outputs. In these conditions, we found - not surprisingly - that effective connectivity between the DG, CA3, and CA1 assessed by linear (GC) and nonlinear (PTE) causality measurements strongly depends on the internal connectivity of the EC. Causality measurements based on partial activity recordings (i.e., from an incomplete set of hippocampal formation nodes) commonly yield unrealistic connectivity patterns. Our work highlights the critical importance of comprehensive neuroanatomical information on the wiring diagram of brain circuits to disambiguate dynamic connectivity patterns since single inhibitory connections or cortical layer interactions may drastically change the effective information flow in the system. However, other constrains of the causality methods need to be also considered and improved. For instance, in this work we have illustrated the impossibility to differentiate, based on standard causality indexes, between excitatory and inhibitory connections, even when the complete wiring diagram is available (as in the built model). In this context, causality acquires different meanings for each case, such as information flow vs. population synchrony for excitatory and inhibitory effective connections, respectively. To better characterize each link is a goal for new methods in the analysis of effective connectivity.

Finally, we have found distinct effective connectivity patterns using GC and PTE methods that will deserve further investigation. In principle, one would expect that PTE should be able to detect interdependency in cases where the GC does not. This is because multivariate GC, which is based in MVAR models, is only sensitive to linear interdependencies, whereas PTE detects, in principle, both linear and nonlinear interdependencies. What we found, instead, is that in the present framework, GC between two time series is sometimes significant whereas the PTE is not. The obtained results can thus be only explained on grounds of their practical applicability. There are two possible reasons. The first one is based on the fact that both indices are parametric, namely, their estimation requires the previous determination of the model order (GC) and the delay and the number of neighbors to estimate the entropy (PTE). We have explored a range of these values and the results do not change significantly with those presented in this study, so we might exclude this possibility. The second one, which is therefore more likely to happen, is that PTE is able to efficiently detect when the connectivity between any two areas in the model is direct rather than indirect, i.e., the information on 1 which can be extracted from 2 is not also present in 3 . For this case, GC would incorrectly detect these indirect connections when the common information is not included in the partializing set.
This hypothesis is supported also by the fact that those cases where the discrepancies are more apparent (i.e., fourth and last columns from the left in Fig. 4) correspond to cases with a bilateral connection between layers LII and LV in the EC, which in our model project to DG and CA1, respectively. This shared information seems to be detected by the PTE, and mask, if any, the true direct one, whereas this is not the case in for the GC. Overall our results highlight the difficulty faced by causality methods when only partial information is available in a network that implements associative loops and long-range inter-areal inhibition.

\section{ACKNOWLEDGMENTS}

C.R.M. and E.P. acknowledge support from the Spanish Ministerio de Economía y Competitividad (MINECO) trough project Nos. TEC2016-80063-C3-3-R and -2-R, respectively. S.C. was supported by MINECO and FEDER funds under Grant No. BFU2015-64380-C2-1-R and European Union Horizon 2020 research and innovation programme under Grant Agreement No. 668863 (SyBil-AA). S.C. acknowledges financial support from the Spanish State Research Agency, through the Severo Ochoa Program for Centres of Excellence in R\&D (SEV- 2013-0317). V.J.L. was supported by a predoctoral fellowship La Caixa-Severo Ochoa from Obra Social La Caixa. F.S.M. acknowledges Brazilian agencies CAPES, CNPq and FAPEAL. F.S.M. and C.R.M. acknowledge CAPES Grant No. PVE 88881.068077/2014-01 for financial support.

${ }^{1}$ H.-J. Park and K. Friston, Science 342, 1238411 (2013).

${ }^{2}$ T. Bliss, G. Collingridge, and R. Morris, "Synaptic plasticity in the hippocampus," in The Hippocampus Book (Oxford University Press, New York, 2007).

${ }^{3}$ R. A. Nicoll and D. Schmitz, Nat. Rev. Neurosci. 6, 863 (2005).

${ }^{4}$ R. C. Malenka and M. F. Bear, Neuron 44, 5 (2004).

${ }^{5}$ J. Rubenstein and M. M. Merzenich, Genes, Brain Behav. 2, 255 (2003).

${ }^{6}$ J. J. Letzkus, S. B. Wolff, and A. Lüthi, Neuron 88, 264 (2015).

${ }^{7}$ K. Kaila, E. Ruusuvuori, P. Seja, J. Voipio, and M. Puskarjov, Curr. Opin. Neurobiol. 26, 34 (2014).

${ }^{8}$ J. Lisman, Curr. Opin. Neurobiol. 22, 537 (2012).

${ }^{9}$ L. A. Atherton, D. Dupret, and J. R. Mellor, Trends Neurosci. 38, 560 (2015).

${ }^{10}$ S. Bouret and S. J. Sara, Trends Neurosci. 28, 574 (2005).

${ }^{11}$ M. E. Hasselmo, Behav. Brain Res. 67, 1 (1995).

${ }^{12}$ M. Helmstaedter, Nat. Methods 10, 501 (2013).

${ }^{13}$ W. Denk and H. Horstmann, PLoS Biol 2, e329 (2004).

${ }^{14}$ S. R. y Cajal, Textura del sistema nervioso del hombre y de los vertebrado (Moya, Madrid, 1899).

${ }^{15}$ R. Lorente de Nó, J. Psychol. Neurol. 46, 113 (1934); available at http:// psycnet.apa.org/index.cfm?fa=search.displayRecord\&UID=1935-01111-001.

${ }^{16} \mathrm{D}$. Amaral and P. Lavenex, "Hippocampal neuroanatomy," in The Hippocampus Book (Oxford University Press, New York, 2007).

${ }^{17}$ D. Marr, D. Willshaw, and B. McNaughton, "Simple memory: A theory for archicortex," in From the Retina to the Neocortex (Springer, Cambridge, 1971), pp. 59-128.

${ }^{18}$ A. Treves and E. T. Rolls, Hippocampus 2, 189 (1992).

${ }^{19}$ A. Treves and E. T. Rolls, Hippocampus 4, 374 (1994).

${ }^{20}$ E. M. Izhikevich, Dynamical Systems in Neuroscience (MIT press, 2007).

${ }^{21}$ E. Izhikevich, IEEE Trans. Neural Networks 14, 1569 (2003).

${ }^{22}$ F. S. Matias, L. L. Gollo, P. V. Carelli, S. L. Bressler, M. Copelli, and C. R. Mirasso, NeuroImage 99, 411 (2014).

${ }^{23}$ V. J. López, E. Pereda, D. Moratal, M. Claudio, and S. Canals, Poster presented at the 10th FENS Forum of Neuroscience, Copenhagen, Denmark (2016).

${ }^{24}$ C. W. Granger, Econometrica 37, 424 (1969).

${ }^{25}$ H. Akaike, IEEE Trans. Autom. Control 19, 716 (1974). 
${ }^{26}$ J. Geweke, J. Am. Stat. Assoc. 77, 304 (1982).

${ }^{27}$ A. K. Seth, J. Neurosci. Methods 186, 262 (2010).

${ }^{28}$ N. Ancona, D. Marinazzo, and S. Stramaglia, Phys. Rev. E 70, 056221 (2004).

${ }^{29}$ Y. Chen, G. Rangarajan, J. Feng, and M. Ding, Phys. Lett. A 324, 26 (2004).

${ }^{30}$ D. Marinazzo, M. Pellicoro, and S. Stramaglia, Phys. Rev. E 73, 066216 (2006).

${ }^{31}$ T. Schreiber, Phys. Rev. Lett. 85, 461 (2000).
${ }^{32}$ G. Niso, R. Bruña, E. Pereda, R. Gutiérrez, R. Bajo, F. Maestú, and F. del Pozo, Neuroinformatics 11, 405 (2013).

${ }^{33}$ T. Schreiber and A. Schmitz, Phys. Rev. Lett. 77, 635 (1996).

${ }^{34}$ A. Sik, M. Penttonen, A. Ylinen, and G. Buzsáki, J. Neurosci. 15, 6651 (1995); available at https://www.ncbi.nlm.nih.gov/pubmed/7472426.

${ }^{35}$ F. Kloosterman, T. Van Haeften, M. P. Witter, and F. H. Lopes da Silva, Eur. J. Neurosci. 18, 3037 (2003).

${ }^{36}$ A. Treves, Hippocampus 14, 539 (2004). 